\title{
Spatial dynamics of a nutrient-phytoplankton system with toxic effect on phytoplankton
}

Chakraborty, Subhendu; Tiwari, P. K.; Misra, A. K.; Chattopadhyay, J.

Published in:

Mathematical Biosciences

Link to article, DOI:

10.1016/j.mbs.2015.03.010

Publication date:

2015

Document Version

Early version, also known as pre-print

Link back to DTU Orbit

Citation (APA):

Chakraborty, S., Tiwari, P. K., Misra, A. K., \& Chattopadhyay, J. (2015). Spatial dynamics of a nutrientphytoplankton system with toxic effect on phytoplankton. Mathematical Biosciences, 264, 94-100. https://doi.org/10.1016/j.mbs.2015.03.010

\section{General rights}

Copyright and moral rights for the publications made accessible in the public portal are retained by the authors and/or other copyright owners and it is a condition of accessing publications that users recognise and abide by the legal requirements associated with these rights.

- Users may download and print one copy of any publication from the public portal for the purpose of private study or research.

- You may not further distribute the material or use it for any profit-making activity or commercial gain

- You may freely distribute the URL identifying the publication in the public portal 


\title{
Spatial dynamics of a nutrient-phytoplankton system with toxic effect on phytoplankton
}

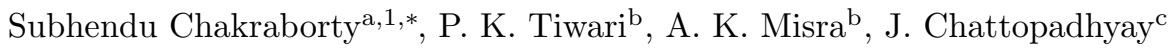 \\ ${ }^{a} I C B M$, University of Oldenburg, Carl von Ossietzky Str. 9-11, D-26129 Oldenburg, Germany \\ ${ }^{b}$ Department of Mathematics, Faculty of Science, Banaras Hindu University, Varanasi 221005, India \\ ${ }^{c}$ AERU, Indian Statistical Institute, 203, B. T. Road, Kolkata 700108, India
}

\begin{abstract}
The production of toxins by some species of phytoplankton is known to have several economic, ecological, and human health impacts. However, the role of toxins on the spatial distribution of phytoplankton is not well understood. In the present study, the spatial dynamics of a nutrient-phytoplankton system with toxic effect on phytoplankton is investigated. We analyze the linear stability of the system and obtain the condition for Turing instability. In the presence of toxic effect, we find that the distribution of nutrient and phytoplankton becomes inhomogeneous in space and results in different patterns, like stripes, spots, and the mixture of them depending on the toxicity level. We also observe that the distribution of nutrient and phytoplankton shows spatiotemporal oscillation for certain toxicity level. Keywords: Nutrient-phytoplankton model, Reaction-diffusion, Toxin, Turing instability, Pattern formation
\end{abstract}

\section{Introduction}

Phytoplankton are the microscopic organisms and form the base of all aquatic food chains. Their presence is important for the large-scale global processes such as the global carbon cycle, oceanatmosphere dynamics and climate change. In natural systems, phytoplankton are known to occur in aggregates or patches which results due to the inhomogeneous distribution of phytoplankton in space [1. The formation of patches alters the optical and acoustical properties of the water column and can have significant impact on the ecological dynamics of marine communities. Several mechanisms have been proposed to describe the patchiness of phytoplankton in the ocean. Some of these mechanisms

\footnotetext{
${ }^{*}$ Corresponding author

Email addresses: subhendu_math@yahoo.co.in (Subhendu Chakraborty), Tel.:+49 441798 3904, Fax: +49 441 7983404 (Subhendu Chakraborty)

${ }^{1}$ Present Address: VKR Centre for Ocean Life, National Institute of Aquatic Resources, DTU Aqua, Technical University of Denmark, Charlottenlund Slot, Jægersborg Allé 1, DK-2920, Charlottenlund, Denmark
} 
can be found in Malchow et al. [2].

On a spatio-temporal scale, the complexity of species interaction is considered as one of the main factors behind the regulation of phytoplankton dynamics [3]. In this respect, toxic chemicals released by some species of phytoplankton (known as toxin producing phytoplankton (TPP)) which are common in most aquatic ecosystems, has the ability to affect species interaction by suppressing the growth and establishment of other species of phytoplankton [4. However, the release of toxic chemicals has other ecological roles, such as precursors for subcellular organelles, cell-wall degradation products, nucleic acid synthesis, nitrogen storage, and defense against predation [5, 6]. Previous studies revealed the importance of toxins affecting other species of phytoplankton in maintaining biodiversity of phytoplankton [7, 8] and also on the bloom dynamics [9]. In spite of such huge importance, there has been little research focused on the role of toxins on the spatial patch formation of phytoplankton.

To the best of our knowledge, there are only few studies showing the occurrence of inhomogeneous biomass distribution of phytoplankton in the presence of TPP due to the spatial movements of plankton. Rao [10] considered a simple TPP-zooplankton system with toxic effect on zooplankton and showed different complex pattern formations depending on the environmental carrying capacity. Chaudhury et al. 11] investigated the effect of toxins released by TPP on grazer zooplankton in a system consisting of two competing phytoplankton species among which one is toxic and one grazer zooplankton. However, they did not consider the effects of toxins on the competing phytoplankton species. Another paper by Roy [12] dealt with two different models; one is a two phytoplankton-one zooplankton system with toxic effect on zooplankton, qualitatively similar in structure with the model of Chaudhury et al. [11, and another one consists of three competing species of phytoplankton among which one is able to release toxic chemicals which negatively affect the growth of the other two species. In both of these studies, abundance of TPP was considered as the representative of the toxic effects. However, in real plankton systems, there is no strict correlation between the toxicity and the abundance of toxic species [13, 14]. Some species, like Phaeocystis, become toxic only when they become abundant. On the other hand, Dinophysis and Alexandrium can be potentially toxic at very low concentrations. Moreover, several other environmental and physical factors also affect the release of toxic chemicals by TPP and accordingly, the rate of the release of toxic chemicals and its toxic effects 
on other species can vary drastically [15, 16, 17]. Therefore, the abundance of TPP cannot always be a perfect representative of the toxic effects on other species of phytoplankton. Here, we attempt to overcome this shortcoming by considering a simple bottom up model without the presence of TPP but with toxic effects on other phytoplankton species. Moreover, algebraically simple models are more suitable for examining the generic behavior of a system [18].

In the present study, we consider the model originally developed by Chakraborty et al. [9] to describe the interaction of a nutrient and a species of phytoplankton with toxic effects on them, applicable to situations where the species is supposed to experience spatial homogeneity. We modify the system by incorporating the effect of spatial movement in the form of diffusion. Our aim is to investigate how the influence of toxic effect affects the spatial distribution of phytoplankton. To do this, we vary the toxic effect on phytoplankton in the presence of spatial movement of nutrient and phytoplankton and explore the distribution of nutrient and phytoplankton in space.

Rest of the paper is organized as follows. In Section 2, we describe the nutrient-phytoplankton (NP) model with toxic effect on phytoplankton in the presence of diffusion. In Section 3, first we analyze the model and state the stability conditions in the absence of diffusion, then analyze the system in the presence of diffusion and find the stability conditions. Specifically, we search for the condition under which Turing instability occurs, where the system without diffusion remains stable, but becomes unstable in the presence of diffusion. In Section 4, we provide numerical evidences of the effects of toxicity on the variation in spatial distribution of nutrient and phytoplankton. Finally, the paper ends with a discussion in Section 5.

\section{Basic model structure}

The spatial extension of the model developed by Chakraborty et al. 9] describing the interaction of a nutrient and a species of phytoplankton can be written as:

$$
\begin{aligned}
\frac{\partial N}{\partial t} & =a-b N P-e N+D_{N}\left(\frac{\partial^{2} N}{\partial x^{2}}+\frac{\partial^{2} N}{\partial y^{2}}\right) \\
\frac{\partial P}{\partial t} & =c N P-d P-\theta \frac{P^{2}}{\mu^{2}+P^{2}}+D_{P}\left(\frac{\partial^{2} P}{\partial x^{2}}+\frac{\partial^{2} P}{\partial y^{2}}\right)
\end{aligned}
$$

where $N(x, y, t)\left(\mathrm{g} \mathrm{C} \mathrm{m}^{-3}\right)$ and $P(x, y, t)\left(\mathrm{g} \mathrm{C} \mathrm{m}^{-3}\right)$, respectively, represent the nutrient concentration and phytoplankton abundance at any time $t$ and location $(x, y)$. Here, $a$ is the rate of constant 
external nutrient input into the system, $b$ is the maximal nutrient uptake rate of phytoplankton, $c$ is the maximal growth rate of phytoplankton, $d$ is the per capita-mortality rate of phytoplankton, $e$ is the per capita-loss rate of nutrient due to sinking from the epilimnion down to the hypolimnion and therefore making these nutrients unavailable for phytoplankton uptake, $\theta$ represents the rate of release of toxic chemicals by the TPP population which measures the strength of toxic effect on phytoplankton population, and $\mu$ denotes the half-saturation constant. Here, the rate of toxin released by the TPP is proportional to the crowding of the phytoplankton and the toxic effect becomes significant when the phytoplankton population reaches high concentration [19]. $D_{N}$ and $D_{P}$ represent the rate coefficients of self-diffusion of $N$ and $P$, respectively. Let, $\Omega$ be the two-dimensional bounded connected square

domain with $\partial \Omega$ as boundary, and $\frac{\partial}{\partial \eta}$ be the outward drawn normal derivative on the boundary. In $\Omega$, we take the following initial conditions for system $(1)$ :

$$
N(x, y, 0)=N_{0}(x, y)>0, P(x, y, 0)=P_{0}(x, y)>0, \forall(x, y) \in \Omega
$$

and the zero-flux boundary conditions

$$
\left.\frac{\partial N}{\partial \eta}\right|_{(x, y)}=\left.\frac{\partial P}{\partial \eta}\right|_{(x, y)}=0, \text { where }(x, y) \in \partial \Omega
$$

It is to be noted here that, the similar system without toxic effect for a homogeneous environment was considered and analyzed by Huppert et al. [20. We also want to mention here that, although our aim was to model the effect of toxins produced by TPP on the spatial distribution of phytoplankton, and according to that we choose our model system, but the term corresponding to the toxic effect can also represent predation on phytoplankton [21]. Therefore, the model can be also used to study the role of predation on the spatial distribution of phytoplankton. However, in the present study, we prefer to stick to the notation of toxic effects, though the model itself is much more general.

\section{Stability analysis}

\subsection{Temporal model and its stability analysis}

First, we perform the stability analysis of the model system (1) in the absence of diffusion. In this case, the system has two non-negative equilibria, namely; trivial equilibrium $E_{1}=\left(\frac{a}{e}, 0\right)$ and interior equilibrium $E^{*}\left(N^{*}, P^{*}\right)$ with $N^{*}=\frac{a}{e+b P^{*}}$, and $P^{*}$ is a positive root of the equation 


$$
b d P^{3}+(b \theta-(a c-d e)) P^{2}+\left(\theta e+b d \mu^{2}\right) P-(a c-d e) \mu^{2}=0
$$

It is clear that the equilibrium $E_{1}$ always exists. Moreover, a unique positive interior equilibrium exists if $0<a c-d e \leq b \theta$.

From the biological point of view, we are mainly interested in studying the stability of the interior equilibrium point $E^{*}$. The Jacobian matrix corresponding to $E^{*}$ can be written as:

$$
J=\left(\begin{array}{ll}
a_{11} & a_{12} \\
a_{21} & a_{22}
\end{array}\right),
$$

where

$$
\begin{aligned}
& a_{11}=-\left(b P^{*}+e\right), \quad a_{12}=-b N^{*} \\
& a_{21}=c P^{*}, \quad a_{22}=\theta \frac{P^{* 2}-\mu^{2}}{\left(\mu^{2}+P^{* 2}\right)^{2}} P^{*} .
\end{aligned}
$$

The corresponding characteristic equation of $J$ is

$$
\lambda^{2}+A \lambda+B=0
$$

where

$$
\begin{aligned}
& A=-\left(a_{11}+a_{22}\right), \\
& B=a_{11} a_{22}-a_{12} a_{21} .
\end{aligned}
$$

Using the Routh-Hurwitz criterion together with the above equation, the following theorem follows immediately:

Theorem 1 The equilibrium point $E^{*}$ of system (1) in the absence of diffusion is locally asymptotically stable iff $A>0$ and $B>0$.

\subsection{Spatiotemporal model and Turing instability}

Now, we study the effect of diffusion on the stability of the system about the interior equilibrium point $E^{*}$. Specifically, we are interested in investigating the Turing instability of the system where the uniform steady state of the system without diffusion is stable, but it is unstable in the partial differential equations with diffusion terms. To study this, first we consider the linearized form of system [1] about $E^{*}\left(N^{*}, P^{*}\right)$ as follows:

$$
\begin{aligned}
& \frac{\partial n}{\partial t}=a_{11} n+a_{12} p+D_{N}\left(\frac{\partial^{2} n}{\partial x^{2}}+\frac{\partial^{2} n}{\partial y^{2}}\right) \\
& \frac{\partial p}{\partial t}=a_{21} n+a_{22} p+D_{P}\left(\frac{\partial^{2} p}{\partial x^{2}}+\frac{\partial^{2} p}{\partial y^{2}}\right)
\end{aligned}
$$


where, $N=N^{*}+n, P=P^{*}+p$. Here, $(n, p)$ are small perturbations of $(N, P)$ about the interior equilibrium point $E^{*}\left(N^{*}, P^{*}\right)$. Let us consider the solution of system (2) in the form

$$
\left(\begin{array}{c}
n \\
p
\end{array}\right)=\left(\begin{array}{c}
n_{k} \\
p_{k}
\end{array}\right) e^{\xi t+i\left(\kappa_{x} x+\kappa_{y} y\right)}
$$

where $\xi$ is the growth rate of perturbation in time $t, \kappa_{x}$ and $\kappa_{y}$ represent the wave numbers of the solutions. The Jacobian matrix of the linearized system can be written as:

$$
\tilde{J}=\left(\begin{array}{cc}
a_{11}-D_{N}\left(\kappa_{x}^{2}+\kappa_{y}^{2}\right) & a_{12} \\
a_{21} & a_{22}-D_{P}\left(\kappa_{x}^{2}+\kappa_{y}^{2}\right)
\end{array}\right) .
$$

In the spatial model, the value of $\xi$ depends on the sum of the square of wave numbers $\kappa_{x}^{2}+\kappa_{y}^{2}[22$. As a result, both wave numbers affect the eigenvalues. This makes clear that some Fourier modes will vanish in the long-term limit whereas others will amplify. For the sake of simplicity, we can make use of $\xi$ being rotational symmetric function on the $\kappa_{x} \kappa_{y}-$ plane and substitute $\kappa^{2}=\kappa_{x}^{2}+\kappa_{y}^{2}$ and obtain the results for the two-dimensional case from the one-dimensional formulation. The corresponding characteristic equation is given by

$$
\xi^{2}+\tilde{A} \xi+\tilde{B}=0
$$

where

$$
\begin{aligned}
& \tilde{A}=A+\kappa^{2}\left(D_{N}+D_{P}\right), \\
& \tilde{B}=B-\left(a_{11} D_{P}+a_{22} D_{N}\right) \kappa^{2}+D_{N} D_{P} \kappa^{4} .
\end{aligned}
$$

Using the Routh-Hurwitz criterion together with the above equation, the following theorem follows immediately:

Theorem 2 The equilibrium point $E^{*}$ is locally asymptotically stable in the presence of diffusion iff $\tilde{A}>0$ and $\tilde{B}>0$.

Clearly, $A>0$ implies $\tilde{A}>0$. Therefore, diffusive instability occurs only when $B>0$, but $\tilde{B}<0$. Hence, the condition for diffusive instability is given by

$$
H\left(k^{2}\right)=D_{N} D_{P} \kappa^{4}-\left(a_{11} D_{P}+a_{22} D_{N}\right) \kappa^{2}+B<0
$$

Here, $H$ is a quadratic function of $\kappa^{2}$ and the graph of $H\left(\kappa^{2}\right)=0$ is a parabola. The minimum of $H\left(\kappa^{2}\right)=0$ is reached at $\kappa^{2}=\kappa_{c}^{2}$, where $\kappa_{c}^{2}$ is given by 
Table 1: The set of fixed parameter values taken within the range prescribed in various previous literature sources (Chaudhuri et al. [1], Edwards 23, and Wang et al. 24])

\begin{tabular}{llll}
\hline Parameter & Definition & Value & Unit \\
\hline$a$ & Constant nutrient inflow & 0.3 & $\mathrm{~g} \mathrm{C} \mathrm{m}^{-3} \mathrm{day}^{-1}$ \\
$b$ & Nutrient uptake rate & 0.5 & $\mathrm{~m}^{3}(\mathrm{~g} \mathrm{C})^{-1} \mathrm{day}^{-1}$ \\
$c$ & Growth rate of $P$ & 0.5 & $\mathrm{~m}^{3}(\mathrm{~g} \mathrm{C})^{-1} \mathrm{day}^{-1}$ \\
$d$ & Mortality rate of $P$ & 0.05 & $\mathrm{day}^{-1}$ \\
$\mu$ & Per capita-loss rate of $N$ & 0.08 & $\mathrm{day}^{-1}$ \\
$D_{N}$ & Half saturation constant & 0.18 & $\mathrm{~g} \mathrm{C} \mathrm{m}^{-3}$ \\
$D_{P}$ & Diffusion rate of $N$ & 0.5 & $\mathrm{~m}^{2} \mathrm{day}^{-1}$ \\
$\theta$ & Diffusion rate of $P$ & 0.01 & $\mathrm{~m}^{2} \mathrm{day}^{-1}$ \\
\hline
\end{tabular}

$$
\kappa_{c}^{2}=\left(a_{11} D_{P}+a_{22} D_{N}\right) / 2 D_{N} D_{P}
$$

Therefore, with the above value of $\kappa_{c}^{2}$, the condition for diffusive instability can be obtained from (4) as

$$
\left(a_{11} D_{P}+a_{22} D_{N}\right)^{2}>4 D_{N} D_{P} B
$$

which is same as

$$
\left(\theta \frac{P^{* 2}-\mu^{2}}{\left(\mu^{2}+P^{* 2}\right)^{2}} P^{*} D_{N}-\left(b P^{*}+e\right) D_{P}\right)^{2}>4 D_{N} D_{P}\left(b c P^{*} N^{*}-\theta \frac{P^{* 2}-\mu^{2}}{\left(\mu^{2}+P^{*}\right)^{2}} P^{*}\left(b P^{*}+e\right)\right)
$$

From the above equation, it is clear that the possibility of diffusive instability increases as $D_{N}$ increases and $D_{P}$ decreases. On the other hand, as we increase $\theta$, L.H.S. of the above inequality apparently increases, whereas the R.H.S. of the inequality decreases which might increase the possibility of diffusive instability. However, since both $N^{*}$ and $P^{*}$ depend on $\theta$, in the next section, we check the dependence of diffusive instability on $\theta$ with the help of numerical simulation. 

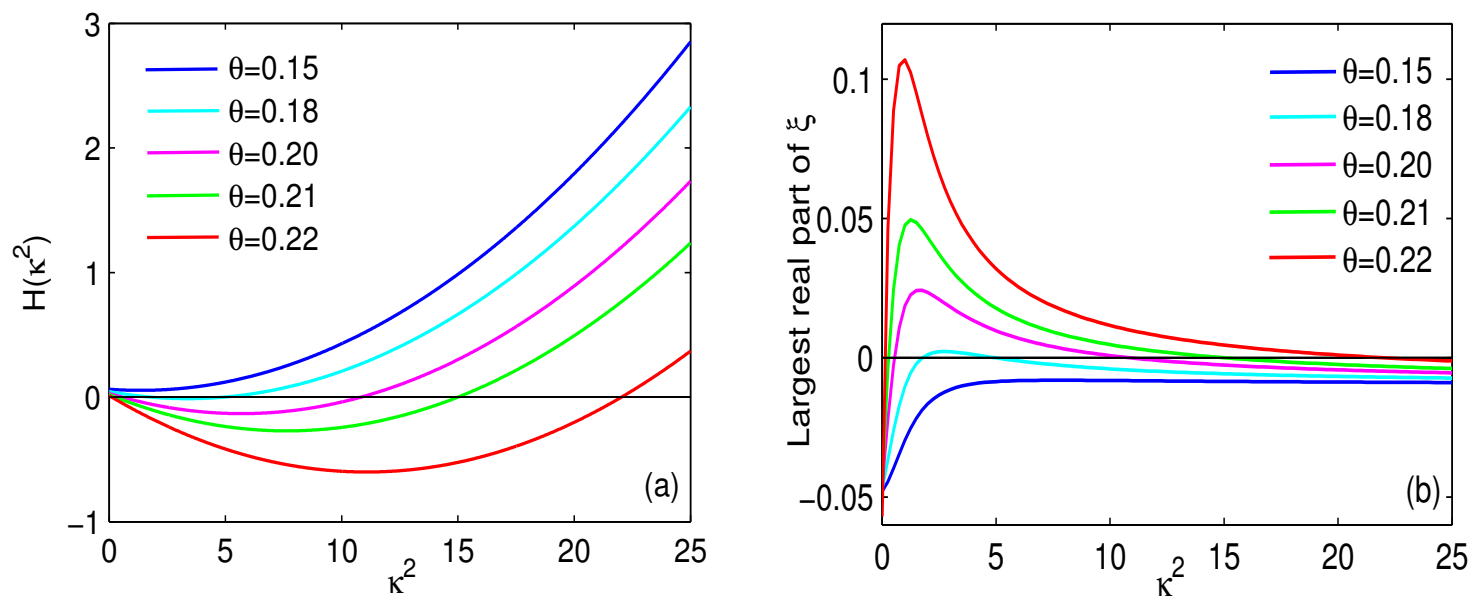

Figure 1: (a) The graph of the function $H\left(\kappa^{2}\right)$ at $\theta=0.15$ (blue), 0.18 (cyan), 0.20 (magenta), 0.21 (green) and 0.22 (red). The other parameter values are taken from Table 1 . An increase in the value of $\theta$ increases the possibility of diffusive instability by increasing the interval of negativity of $H\left(\kappa^{2}\right)$. (b) Dispersan relation plotting the largest real part of the eigenvalues at different $\theta: \theta=0.15$ (blue), 0.18 (cyan), 0.20 (magenta), 0.21 (green) and 0.22 (red).

\section{Numerical simulation}

For our numerical simulation, the parameter values are taken from the range described in different literature sources and given in Table 1. In natural systems, diffusion of phytoplankton can be very low as well as very high; even it can be smaller as well as higher than that of the nutrient because of the viscosity and living of phytoplankton [24, 25. In the present study, we have assumed a lower diffusitivity of phytoplankton than that of the nutrient.

First of all, we look at the plots of $H\left(\kappa^{2}\right)$ (Eq. 44) for different values of $\theta$. This will give us an idea about the occurrence of Turing instability and the range of $\theta$ for which it exists. Turing instability condition $\min \left(H\left(\kappa^{2}\right)\right)<0$ is satisfied for $\theta \geq 0.1762$. In Figure 1(a), we plot the curve $H\left(k^{2}\right)=0$ for $\theta=0.15$ (blue), 0.18 (cyan), 0.20 (magenta), 0.21 (green) and 0.22 (red). It is clear that the interval for $\min \left(H\left(\kappa^{2}\right)\right)<0$ increases with increase in $\theta$. Therefore, we can expect an increase in the possibility of Turing instability with an increase in $\theta$. We also plot the real part of the largest eigenvalue of the characteristic equation (3) (Figure 1(b)) of system (1) for the values of $\theta$ as considered in Figure 1(a). The length of the interval of $\kappa^{2}$ within which the largest real part of the eigenvalues is positive (i.e., for which the system with diffusion is unstable) increases with increasing magnitude of $\theta$.

Next, we observe the local asymptotic stability and Turing instability of system (1) depending on 


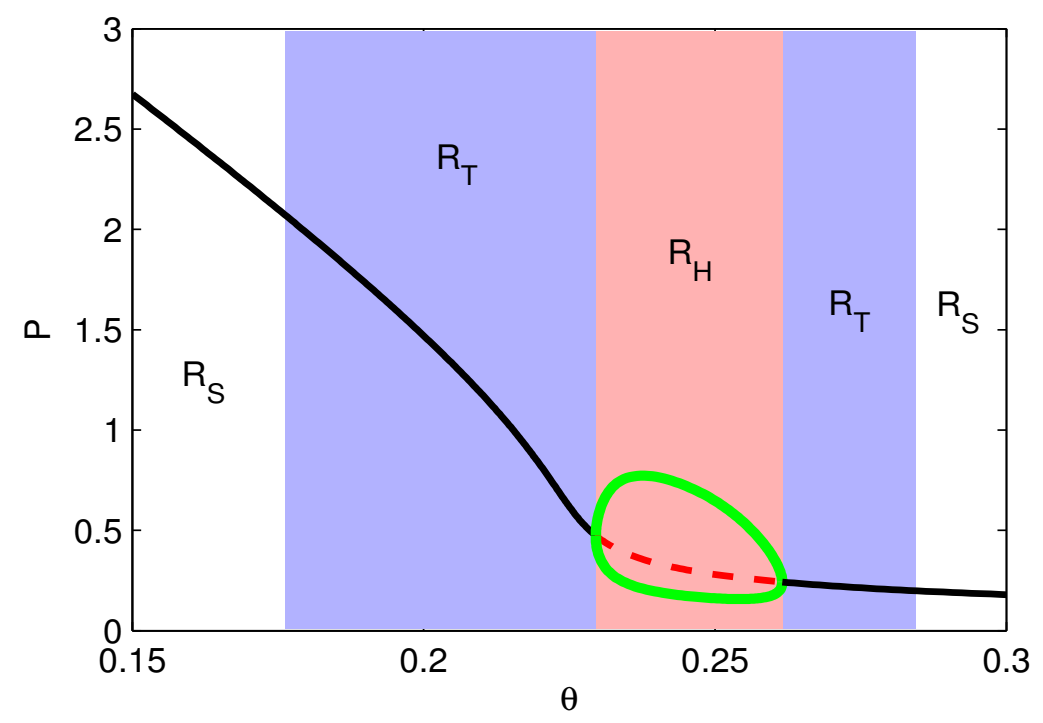

Figure 2: Variations in the steady-state values of $P$ as $\theta$ changes where the other parameter values are the same as in Table 1 The black line represents the stable steady state, red dashed line represent the unstable steady state and the green line indicates the maximum and minimum values of the stable limit cycle. $R_{S}$ (white), $R_{T}$ (blue) and $R_{H}$ (pink) are ranges of $\theta$ indicating stable steady state, Turing instability and Turing-Hopf domains, respectively.

the toxic effect, $\theta$. Figure 2 shows a one-dimensional bifurcation diagram by varying $\theta$ which gives an overview of how phytoplankton abundance changes and different dynamics occur with variation in $\theta$. Specifically, we plot the steady-state values of the abundances of phytoplankton with $\theta$. The black and red (dashed) lines indicate that the steady state is stable and unstable, respectively. Additionally, the green lines represent the maximum and minimum abundances of the populations for the stable limit cycle. Clearly, for lower values of $\theta(\theta<0.1762)$, system (1) always remains stable and the corresponding range of $\theta\left(R_{S}\right)$ is indicated by the white color. As we increase $\theta$, for certain range $R_{T}$, marked by the blue color, we find Turing instability, i.e., where system (1) becomes unstable and can show pattern formation in space in the presence of diffusion whereas the system without diffusion still remains stable. Clearly, in these two regions, increase in toxic effect $\theta$ results in the decrease in phytoplankton abundance. Further increase in $\theta$ results in Hopf-bifurcation and the system shows a limit cycle oscillation for $\theta \in(0.2295,0.2615)$. The corresponding range $R_{H}$ of $\theta$ for limit cycle oscillation is marked in the figure with pink color. However, higher values of $\theta$ make the system without diffusion stable with very low phytoplankton abundances where for certain range $(\theta \in(0.26154,0.2844))$ again Turing instability occurs. 


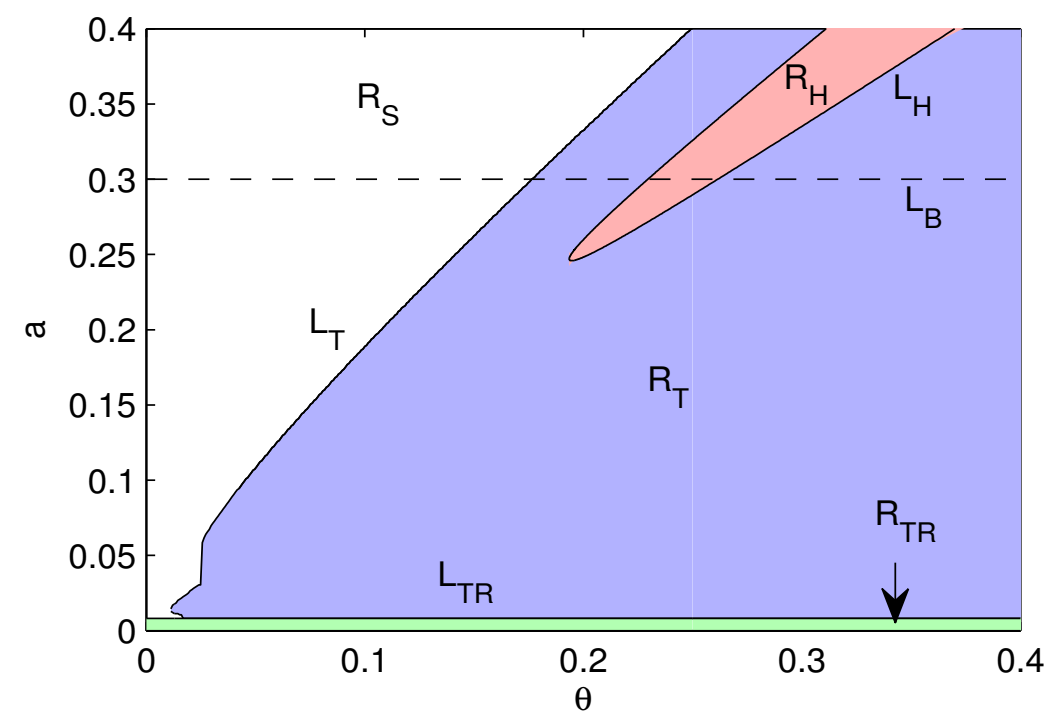

Figure 3: Two-parameter bifurcation diagram as a function of $\theta$ and $a$. Regions $R_{S}$ (white), $R_{T}$ (blue), $R_{H}$ (pink) and $R_{T R}$ (green) are corresponding to the stable steady state, Turing instability, Turing-Hopf domains and extinction of phytoplankton, respectively.

Since nutrient inflow in the plankton system plays an important role in determining the dynamics of the system, we observe how different dynamics observed in Figure 2 change with nutrient inflow, $a$, together with the toxic effect, $\theta$. In Figure 3 , we draw a two-parameter bifurcation diagram by varying $a$ (along x-axis) and $\theta$ (along y-axis). Color codings and notations of different regions in Figure 3 are same as that of Figure 2. Additionally, in this case we find another region $R_{T R}$ for low values of $a$ (marked by green color) where the phytoplankton population cannot survive. Here, the lines $L_{T R}$, $L_{H}$ and $L_{T}$ are corresponding to the transcritical bifurcation, Hopf-bifurcation, and Turing curve, respectively. Moreover, the dashed line, $L_{B}$, is the one along which we plotted the one dimensional bifurcation diagram shown in Figure 2 . From Figure 3 it is clear that there is no spatial inhomogeneity in the system in the absence of toxic effect. However, the increase in toxic effect can result in Turing instability where the spatial distribution of phytoplankton becomes inhomogeneous. Moreover, high nutrient input needs high level of toxic effect for phytoplankton to become inhomogeneous in space. Furthermore, high nutrient input can also make the system unstable and import oscillation in the phytoplankton population for certain range of toxic effect.

In the following, we will show the formation of different patterns due to the variation of toxic effect, $\theta$. 


\subsection{Pattern formation}

Here, we perform extensive numerical simulations of the spatial model system (1) in two dimensional space using the forward finite difference method, and the qualitative results of different pattern formations due to the variation in toxic effect $\theta$ are shown. The set of parameter values used for the numerical simulation are same as given in Table 1 unless it is mentioned.

To analyze the dynamic behavior of system (1), we plot the stationary distribution of nutrient and phytoplankton in two-dimensional space. Here, the system is studied on a rectangular spatial grid of $50 \times 50$ points (except Figure 6 which is studied on $100 \times 100$ points) with the Neumann boundary conditions and run the simulation up to the time $t=5000$ for different values of $\theta$. The space step is taken as 0.2 , and the time step as 0.005 . It is assumed that the nutrient and phytoplankton are spread over the whole domain at the beginning. We know that the spatial dynamics of a system are greatly affected by the choice of the initial conditions. If the initial spatial distribution of nutrient and phytoplankton is homogeneous, then the species distribution remains homogeneous forever, which is not so interesting [26]. Apart from that, from a biological point of view, it is reasonable to consider a scattered non-uniform initial distribution of species over the space under consideration. Here, such scattered initial distribution has been employed by considering a random sampling of nutrient and phytoplankton abundance around the equilibrium values of the corresponding non-spatial model. It is to be noted here that, the time at which we stopped the simulations is sufficient to assume that the patterns attained the stationary state and they do not change further with time.

In Figure 4, we plot the spatial distribution of nutrient (first column) and phytoplankton (second column), respectively, where the first, second, third and fourth rows represent the dynamics at $\theta=0.19$, $\theta=0.20, \theta=0.22$ and $\theta=0.26$, respectively. It is observed that the distribution of nutrient and phytoplankton remains homogeneous in space in the absence of toxic effect (figure is not shown). However, the presence of toxic effect makes the distribution inhomogeneous in space and results in different types of Turing patterns with variation in toxic effect $\theta$. Clearly, at $\theta=0.19$, we find the mixture of cold spot and stripe patterns for phytoplankton distribution. However, at $\theta=0.20$, we observe a stripe pattern. Again, further increase in $\theta(\theta=0.22)$ results the mixture of hot spot and stripe patterns. Finally, at $\theta=0.26$, we get stable hot spots with high phytoplankton densities in 

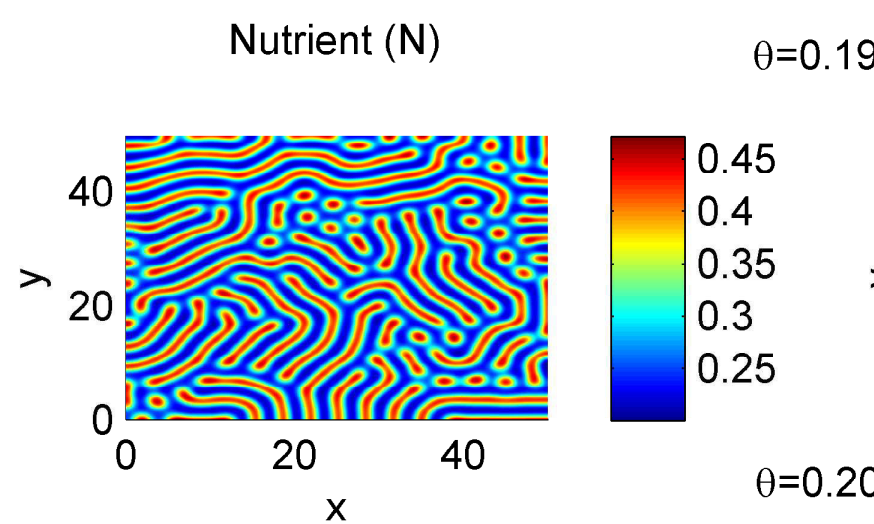

19 Phytoplankton (P)
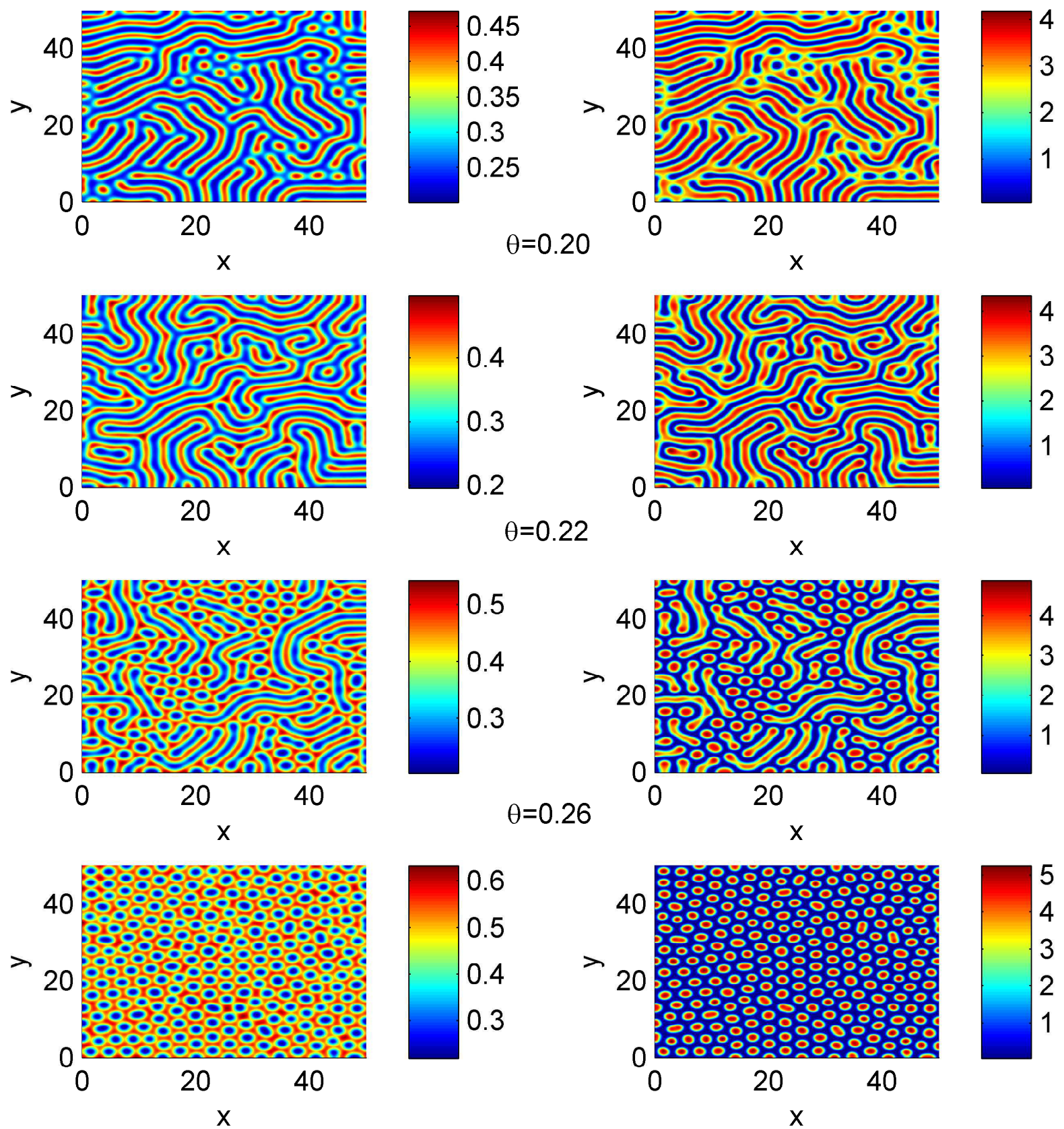

Figure 4: Stationary nutrient (first column) and phytoplankton (second column) distribution over space at different values of $\theta: \theta=0.19$ (first row), $\theta=0.20$ (second row), $\theta=0.22$ (third row) and $\theta=0.26$ (fourth row). 

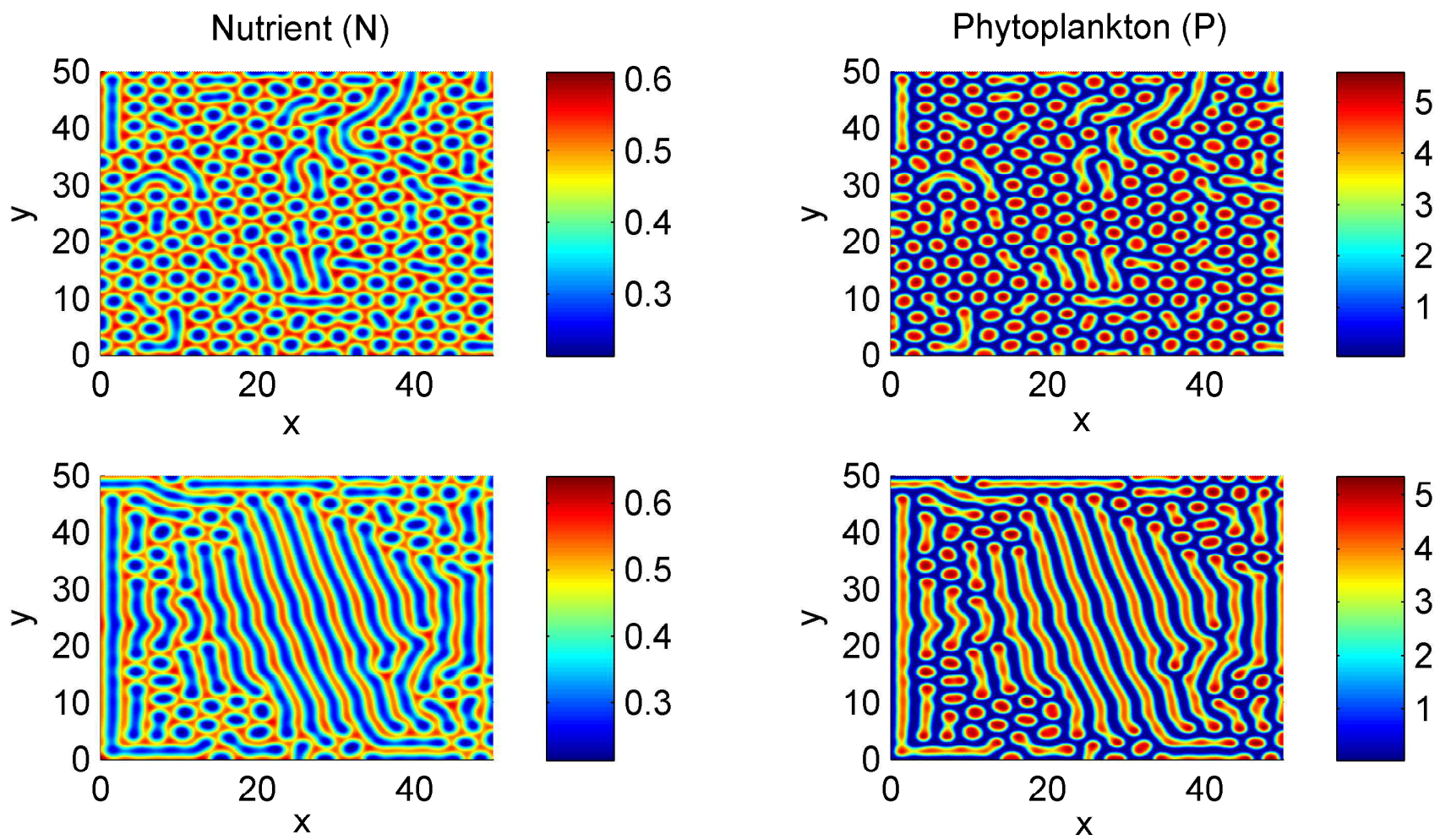

Figure 5: Stationary nutrient (first column) and phytoplankton (second column) distribution over space starting with different initial distribution of nutrient and phytoplankton: random sampling of nutrient and phytoplankton abundance around the equilibrium values of the corresponding non-spatial model (first row), and initial distribution mentioned in Eq. 6 (second row). Parameter values used: $\theta=0.275, a=0.35$ and the other parameter values are same as in Table 1

isolated zones. It is to be mentioned here that the hot spot pattern for the last case is observed for parameters lying in the Turing-Hopf domain. Moreover, since a high abundance of phytoplankton keeps the nutrient concentration low, hot spots in the spatial structure of phytoplankton population correspond to cold spots in the case of the spatial distribution of nutrient.

Next, we examine the spatial pattern formation of system (1) for $\theta=0.275$ and $a=0.35$. This set of parameter values lies in the Turing-Hopf domain where the homogeneous steady state of the system without diffusion is unstable. In the previous figure, we see that the consideration of parameter set from this region makes phytoplankton distribution stationary. Here, we will show how such patch formation differs depending on the initial distribution of nutrient and phytoplankton although the distributions ultimately reach to a stationary state at the long time. In Figure 5 , we start with two different initial distributions: random sampling of nutrient and phytoplankton abundance around the 

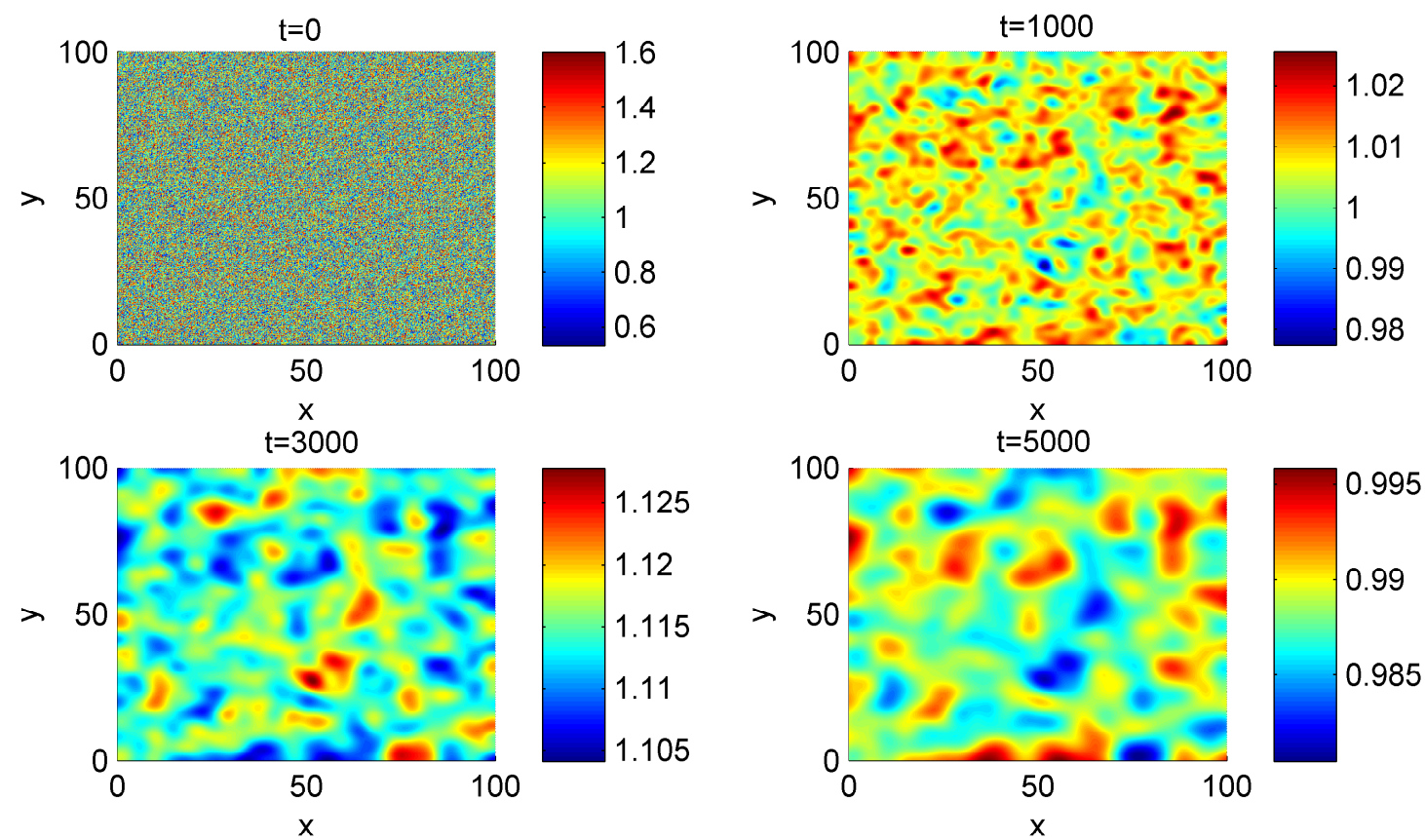

Figure 6: Spatiotemporal pattern exhibited by phytoplankton population at different time steps: $t=0, t=1000$, $t=3000, t=5000$. Parameter values used: $\theta=0.2, a=0.25, D_{N}=0.005, D_{P}=0.002$ and the other parameter values are same as in Table 1

equilibrium values of the corresponding non-spatial model (first row), and

$$
\begin{aligned}
& N(x, y, 0)=N^{*}-\varepsilon_{1}(x-25), \\
& P(x, y, 0)=P^{*}-\varepsilon_{2}(y-25),
\end{aligned}
$$

where $\epsilon_{1}=0.02$ and $\epsilon_{2}=0.003$ (second row). Clearly, the stationary states show different patterns for different initial distributions of nutrient and phytoplankton. Although, in both the cases, phytoplankton distribution shows the mixture of the hot spot and stripes, but, hot spots are dominant when initial random distributions are considered, whereas stripes are dominant in the other case.

We also find that for certain parametric setup within Turing-Hopf domain, the distributions of nutrient and phytoplankton do not converge to any stationary steady state. We start with the random sampling of nutrient and phytoplankton abundance around the equilibrium values of the corresponding non-spatial model. Figure 6 shows that the distribution of phytoplankton population does not converge to any stationary state where the corresponding time steps $(t=0,1000,2000,3000)$ are mentioned in the caption of figures and associated colorbars represent the fluctuations of phytoplankton population densities at different time steps. Clearly, the observed patterns are completely different from the ones 

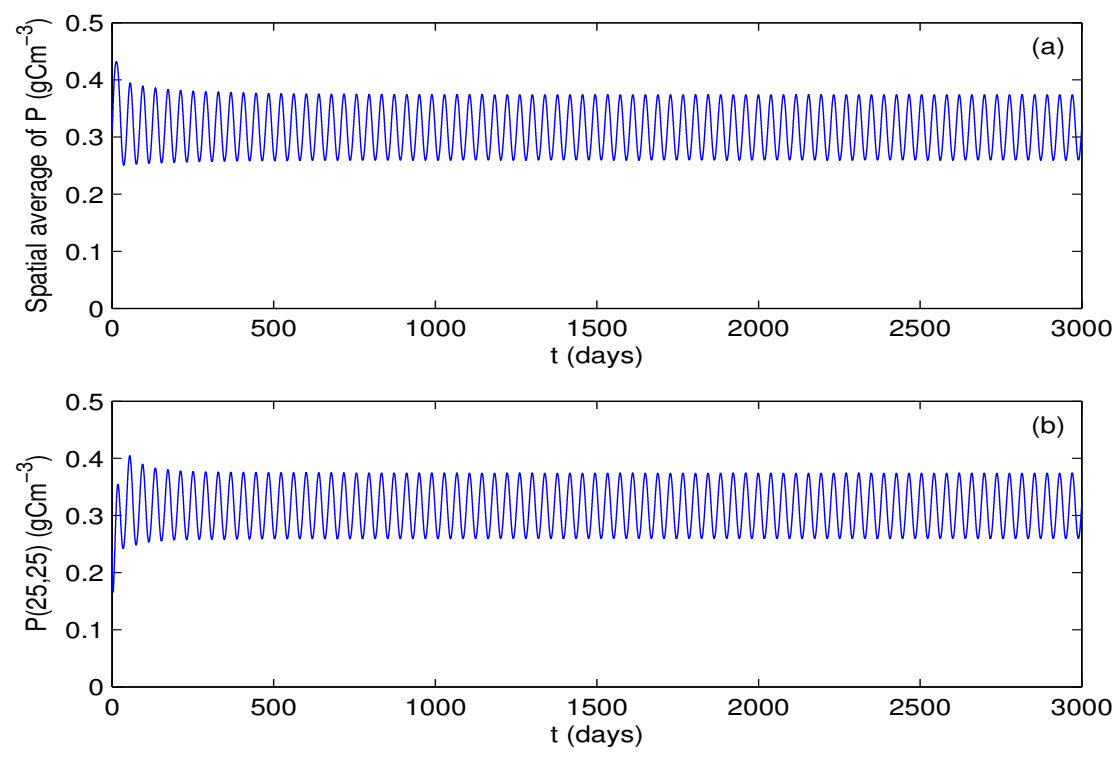

Figure 7: (a) Plot of spatial average values of phytoplankton population against time showing limit cycle oscillation. (b) Time evolution of phytoplankton population at the spatial location $(25,25)$. Parameter values are same as in Figure 6

observed in the previous cases. The spatial average of phytoplankton population with time is plotted in Figure 7(a) which clearly shows limit cycle oscillation. We also plot the phytoplankton abundance at the spatial position $(25,25)$ with respect to time in Figure 7 (b) which also shows a regular oscillation with time.

\section{Discussion}

The production of toxins by some species of phytoplankton (TPP) is common in many aquatic ecosystems. There are studies revealing the importance of toxic effects on other phytoplankton and grazer zooplankton on plankton dynamics [27, 28, 29]. Some of the previous studies discussed about the role of toxins in maintaining coexistence and biodiversity in plankton systems [7, 8] and affecting the bloom dynamics [9]. However, the effects of toxins on the spatial distribution of phytoplankton are not properly investigated. In the present paper, we demonstrate how an inhomogeneous biomass distribution of phytoplankton emerges over space due to the toxic effect. The study shows that, in the absence of toxic effects, the distribution of phytoplankton remains homogeneous in spite of spatial movements of nutrient and phytoplankton. However, the presence of toxic effect makes phytoplankton distribution inhomogeneous in space and generates different types of Turing patterns depending on the strength of toxic effects and initial distribution of nutrient and phytoplankton population. We 
also find situations when the distribution of phytoplankton does not converge to any stationary state, instead oscillates with time.

Turing instability means, for certain values of the diffusion coefficients of nutrient and phytoplankton, a uniform, steady-state solution of the system, which is stable in the absence of diffusion, becomes unstable when diffusion is incorporated. To investigate this, one can give a small perturbation around the uniform steady-state solutions of the system and determine the conditions under which these fluctuations increase. From our result, it can be shown that instability can't occur if the fluctuations have very large or very small length-scales (wavelengths); fluctuations of the intermediate-size scale can only result Turing behavior which is a very common and expected result [30. Very large fluctuations are approximately uniform and not so effective to upset the already uniform stable equilibrium. On the other hand, very small scale fluctuations are quickly dissipated by diffusion before they grow. The range of the intermediate-size fluctuations where nonuniformities can develop, is mainly dependent on both the physical environment (e.g., diffusivities) and some of the biological parameters. Our study shows that, diffusion and high toxic effect on phytoplankton keeps the size scales of the fluctuation in the critical intermediate range that leads to Turing behavior.

Previously, there are only few studies demonstrating the role of toxins in the pattern formation in plankton systems [10, 11, 12]. They found that the presence of toxic species can import inhomogeneity in plankton system. However, all of the previous studies considered that the toxic effects are significant only when the abundance of the toxic species is relatively high and toxic effects are proportional to the toxic species abundance. In this respect, our study is different from the previous ones. As we know that, in natural aquatic systems, toxic species can have significant variation in the toxic effects due to the changes in environmental and physical conditions [15, 16, 17] and the toxin production is not always related to high TPP abundance [13, 14, thus, the toxic species abundance cannot always be the exact representative of the toxic effects. Accordingly, in the present study, we have considered a simple nutrient-phytoplankton model with toxic effects without explicitly considering the presence of toxic phytoplankton. Interestingly, in this more general, but very simple model, we have found an important role of toxic effects on the spatial distribution of phytoplankton. Additionally, we have found distinctively different spatial structures for the distribution of nutrient and phytoplankton with 
the variation in toxicity level.

Spatial variability of phytoplankton is very common in aquatic ecosystems. Several attempts have been made to explain such spatial variation of phytoplankton using different types of mathematical models [3, 12. However, only a few of them considered nutrient-phytoplankton (NP) models. Although, some examples can be found for the consideration of reaction-advection-diffusion equations based on NP models to explain the spatial variation of phytoplankton [24, 31, 32, the consideration of only reaction-diffusion equations are very rare. Ruan 33 incorporated diffusion in a NP system with a delay in the growth response of the plankton to nutrient uptake and a distributed delay for the nutrient recycling. He has found diffusion-driven instability of the reaction-diffusion system and a family of traveling waves in the presence of delay. Further, Gourley and Ruan [34] examined the role of time delay with a weighted spatial averaging to account for the movement of individuals during the time delay period on a diffusive NP model and found a homogenizing role of the averaging. In the present study, we have also investigated a NP system with diffusion, but our aim was completely different; we have examined the role of toxic effect on the spatial distribution of phytoplankton.

In conclusion, we can say that, toxins produced by TPP have a deep impact on plankton systems. It can drastically change the system dynamics by changing the spatial distribution of phytoplankton. To further extrapolate the results of this study, further extension of this model system might be worth investigating. Firstly, we can extend the current model by incorporating zooplankton population in the system with an extra mortality of zooplankton due to the toxic effect without explicitly considering the presence of toxic species since zooplankton grazing plays an important role in plankton dynamics. Secondly, advection plays an important role in the spatial movement of plankton in the oceans. Thus, it would also be interesting to examine the role of toxic effects in a plankton system which is addressed using a pair of reaction-advection-diffusion equations.

Acknowledgment: S.C wishes to thank the Alexander von Humboldt Foundation for financial support in the form of a postdoctoral fellowship at the ICBM, Carl von Ossietzky University, Oldenburg, Germany. PKT acknowledges the University Grant Commission, New Delhi, India, for the financial assistance in the form of Senior Research Fellowship (19-12/2010(i)EU-IV). 


\section{References}

[1] Brentnall, S.J., Richards, K.J. Brindley, J., Murphy, E.: Plankton patchiness and its effect on larger-scale productivity. J. Plank. Res. 25, 121-140 ( 2003)

[2] Malchow, H., Petrovskii, S., Venturino, E.: Spatiotemporal patterns in Ecology and Epidemiology: Theory, Models, Simulations. Chapman \& Hall / CRC Press (2008)

[3] Medvinsky, A., Petrovskii, S., Tikhonova, I., Malchow, H., Li, B.: Spatio-temporal complexity of plankton and fish dynamics in simple model ecosystems. SIAM Rev. 44(3), 311-370 (2002)

[4] Hallam, T., Clark, C., Jordan, G.: Effects of toxicants on populations: a qualitative approach. II. First order kinetics. J. Theor. Biol. 18, 25-37 (1983)

[5] Guisande, C., Frangopulos, M., Maneiro, I.,, Vergara A.R., Riveiro, I.: Ecological advantages of toxin production by the dinoflagellate Alexandrium minutum under phosphorus limitation. Mar. Ecol. Prog. Ser. 225, 169-176 (2002)

[6] Turner, J.T., Tester, P.A.: Zooplankton feeding ecology: copepod grazing during an expatriate red tide. In: Cosper, E.M. (Ed.), Novel Phytoplankton Blooms: Causes and Impacts of Recurrent Brown Tides and Other Unusual Blooms. Springer-Verlag, Berlin, 359-374 ( 1989)

[7] Roy, S., Chattopadhyay, J.: Toxin-alleopathy among phytoplankton species prevents competition exclusion. J. Biol. Syst. 15(1), 73-93 (2007)

[8] Roy, S.: Do phytoplankton communities evolve through a self-regulatory abundance diversity relationship? BioSystems 95, 160-165 (2009)

[9] Chakraborty, S., Chatterjee, S., Venturino, E., Chattopadhyay, J.: Recurring plankton bloom dynamics modeled via toxin producing phytoplankton. J. Biol. Phys. 33, 271-290 (2007)

[10] Rao, F.: Spatiotemporal dynamics in a reaction-diffusion toxic-phytoplankton-zooplankton model. J. Stat. Mech. Theory Exp. Article ID 08014 (2013)

[11] Chaudhuri, S., Chattopadhyay, J., Venturino, E.: Toxic phytoplankton-induced spatiotemporal patterns. J. Biol. Phys. 38, 331-348 (2012) 
[12] Roy, S.: Spatial interaction among nontoxic phytoplankton, toxic phytoplankton, and zooplankton: emergence in space and time. J. Biol. Phys. 34, 459-474 (2008)

[13] Bushati, M., Koni, E., Miho, A., Bregaj, M.: Temporal distribution of potentially toxic algae (dinoflagelates and diatoms) in Butrinti Lagoon. Natura. Montenegrina. Podgorica 9(3), 307-319 (2010)

[14] Graneli, E., Johansson, N.: Increase in the production of allelopathic Prymnesium parvum cells grown under N- or P-deficient conditions. Harmful Algae 2, 135-145 (2003)

[15] Chakraborty, S., Roy, S., Chattopadhyay, J.: Nutrient-limited toxin production and the dynamics of two phytoplankton in culture media: A mathematical model. Ecol. Model. 213(2), 191-201 (2008)

[16] Johansson, N., Graneli, E.: Influence of different nutrient conditions on cell density, chemical composition and toxicity of Prymnesium parvum (Haptophyta) in semi-continuous cultures. J. Exp. Mar. Biol. Ecol. 239, 243-258 (1999)

[17] Shilo, M.: Toxins of Chrysophycae. In: Kadis, S., Ciegler, A., Ajl, S.J. (Eds.), Microbial Toxins. Algal and Fungal Toxins 7, Academic Press, New York, 67-103 (1971)

[18] Scheffer, M.: Fish and nutrients interplay determines algal biomass: a minimal model. Oikos 62, 271-282 (1991)

[19] Vardi, A., Schatz, D., Beeri, K., Motro, U., Sukenik, A., Levine, A., Kaplan, A.: Dinoflagellatecyanobacterium communication may determine the composition of phytoplankton assemblage in a mesotrophic lake. Current Biology 12(20), 1767-1772 (2002)

[20] Huppert, A., Blasius, B., Stone, L.: A model of phytoplankton blooms. Am. Nat. 159, 156-171 $(2002)$

[21] Chakraborty, S., Feudel, U.: Harmful algal blooms: combining excitability and competition. Theo. Ecol. 7(3), 221-237 (2014)

[22] Baurmann, M.: Turing instabilities and pattern formation in a benthic nutrient-microorganism system. Math. Biosci. Eng. 1, 111-130 (2004) 
[23] Edwards, A.M.: Adding detritus to a nutrient-phytoplankton-zooplankton model: a dynamicalsystems approach. J. Plank. Res. 23(4), 389-413 (2001)

[24] Wang, W., Zhao, M., Dai, C., Pan, X.: Nonlinear dynamics of a nutrient-plankton model. Abstract and Applied Analysis 2014, Article ID 451757, 1-10 (2014)

[25] Powell T., Richerson P.: Temporal variation, spatial heterogeneity, and competition for resources in plankton systems: A theoretical model. Am. Nat. 125, 431-464 (1989)

[26] Petrovskii, S.V., Malchow, H.: A minimal model for pattern formation in a prey-predator system. Math. Comput. Model. 29, 49-63 (1999)

[27] Chakraborty, S., Bhattacharya, S., Feudel, U., Chattopadhyay, J.: The role of avoidance by zooplankton for survival and dominance of toxic phytoplankton. Ecol. Complex. 11, 144-153 (2012)

[28] Chatterjee, S., Venturino, E., Chakraborty, S., Chattopadhyay, J.: A simple mathematical model for seasonal planktonic blooms. Math. Meth. Appl. Sci. 16, 1738-1750 (2009).

[29] Hulot, F.D., Huisman, J.: Allelopathic interactions between phytoplankton species: the roles of heterotrophic bacteria and mixing intensity. Limno. Oceano. 49, 1424-1434 (2004)

[30] Okubo, A.: Diffusion and ecological problems: mathematical models. Biomathematics 10, Springer-Verlag, New York (1980)

[31] Serizawa, H., Amemiya, T., Itoh, K.: Patchiness in a minimal nutrient-phytoplankton model. J. Biosc. 33(3), 391-403 (2008)

[32] Arinoa, O., Boushabab, K., Boussouar, A.: A mathematical model of the dynamics of the phytoplankton-nutrient system. Nonlinear Analysis: RWA 1, 69-87 (2000)

[33] Ruan, S.: Turing instability and travelling waves in diffusive plankton models with delayed nutrient recycling. IMA J. Appl. Math. 61, 15-32 (1998)

[34] Gourley, S.A., Ruan, S.: Spatio-temporal delays in a nutrient-plankton model on a finite domain: linear stability and bifurcations. Appl. Math. Comp. 145, 391-412 (2003) 